\title{
The Gyrostatic Force of Rotary Engines*
}

\author{
Its Nature and Significance for Aviation
}

\author{
By Albert Kapteyn, President of the Aviation Section of the Dutch Aero Club
}

Grooststic action is more familiar in name than in principle to the majority of laymen, and even those who have had some training in mechanics often seem to Lave but a superficial and sometimes inaccurate conception of the force in question. It is, however, a subject that ought to be thoroughly well understood by everyone associated with the use of rotating masses, and, above all others, pilots of aeroplanes ought to be thoroughly au fait with its action, because the flying machine affords greater possibilities for its demonsira-

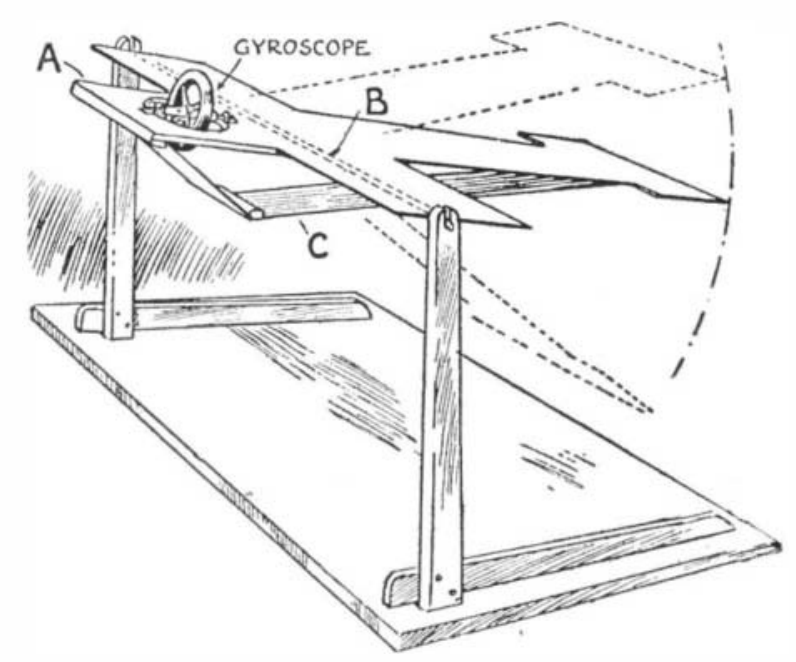

Fig. 1. - Sketch lllustrating a Simple Model That Can be Used to Demonstrate the Effect of Gyrostatic Force on the Control of an Aeroplane.

tion than almost any other conceivable apparatus. The reason for this is obvious when we consider the nature of gyrostatic force. It is a force that comes into play when the axis or shaft of a rotating body by itself rocked from its original position. Take, for example, the propeller-shaft of a flying machine. So long as the machine proceeds in an absolutely unswerving the machine proceeds in an absolutely unswerving
path, the gyrostatic force of the rotating propeller, or path, the gyrostatic force of the rotating propeller, or
the rotary engine if such happens to be used, remain dormant. But, for how long does an aeroplane follow an absolutely unswerving path? Scarcely for a second of time. Almost every instant it is being steered either to the right or to the left, and often it pitches in its line of flight. Every such motion rocks the axis of the propeller-shaft and brings into life the axis of the propeller-shaft and brings into life the dormant gyrostatic force, the magnitude of which de-
pends on the rapidity with which this rocking motion takes place. It is therefore obvious that the gyrostatic force will be most in evidence when the pilot is at tempting to execute a very quick maneuver, such as turning about in a very short circle.

It is notorious that several fatal accidents have taken place recently under circumstances of this sort. Feeling convinced, therefore, that gyrostatic force is sufficient to cause dangerous interference with the sufficient to cause dangerous interference with the
control, and having found that many aviators are sufcontrol, and having found that many aviators are suf-
ficiently acquainted with the principles of gyrostatic action to really enable them to appreciate when some difficulties, that they have attributed to the weather,

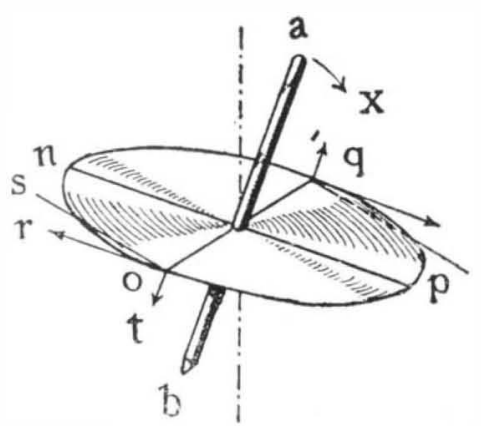

Fig. 2.-Diagrammatic Sketch Illustrating the Nature and Direction of the Gyrostatic Forces in a Spin ning Disk.

might in reality have been due to this very force, I have thought it well to endeavor to treat the subject hereunder in simple but nevertheless, I hope, con vincing terms. There is, moreover, all the more reason to treat the matter scientifically at this juncture, because nothing is so calculated to raise scepticism in the minds of those who do not know, than the very erroneous conclusions that have of late been ad vanced by some of those who profess to recognize the dangers of this very force. Some people seem to think, for instance, that gyrostatic action may be the direct means of fracturing main spars, struts, tie wires, or other constructional members that are not in any way directly related to the axis of the revolving shaft. It may be said at once that so far as any direct effect of gyrostatic action is concerned, the only constructional damage it is likely to do is to bend or break the propeller-shaft, and it is essentially my purpose in thi article not so much to write a warning on this aspect of the case as to point out that the immediate consequence of gyrostatic action is interference with control.

The outstanding feature of gyrostatic reaction is that it takes place in a plane at right angles to that of the applied force and also to that of the axis of rotation of the shaft. Thus, for example, in a steam ship pitching through the waves the gyrostatic reaction from a turbine shaft placed fore and aft exerts a pressure on the sides of the bearings, not on the tops and bottoms as might be supposed, and as would in fact be the case were the shaft at rest and only its ordinary inertia to be taken into account. Similarly, in an aeroplane, if it pitches in its line of flight the in an aeroplane, if it pitches in its line of flight the
gyrostatic reaction tends to upset the steering, and conversely if it is steered off a straight path the gyrosatic reaction tends to make it pitch. The question as to whether the nose of the machine will tilt or dip depends on the direction of rotation of the motor and the direction in which it is steered. Suppose the aeroplane to be a monoplane with a tractor screw and that the motor has a clock-wise rotation viewed from in front; then steering to the left, $i$. e. keeping the pylone on the left hand, will tend to make the machine dive. If the same engine is placed on a biplane so that the propeller is behind. The rotation of the shaft will have been reversed in respect to the direction of turning, consequently the machine will tend to tilt during the same maneuver.

It will be instructive to see what magnitude such forces take with machines as they may be actually constructed. For example, let us suppose that we have a powerful rotary motor, weighing 100 kilogram (220.5 pounds), driving a propeller weighing 10 kilograms (22.05 pounds) at 1,200 revolutions per minute; let us further suppose that the radius of the gyration of the motor is 0.33 meter (one foot). and that of the propeller 0.83 meter ( 2.72 feet). The gyrostatic reac tion will have a magnitude depending on the rapidity with which the course of the aeroplane is altered in wight. We will imagine that the pilot completes an flight. We will imagine that the pilot completes an
angle of 90 degrees, or in other words, changes to a line of flight at right angles to his original path, in three seconds. Wilbur Wright, it may be mentioned, frequently made such an angle in one second and less. Applying these values, the gyrostatic couple produced by the motor is 73.5 kilogram meters (531.6 foot pounds.), and that produced by the propeller is 42.4 kilogram meters (306.7 foot pounds), the com bined effect being thus 116 kilogram meters ( 839 foot pounds). This force is a torque tending to make the machine dive or tilt as the case may be, and the principal means at the disposal of the pilot for resisting, the action is the elevator. If we suppose that this member is situated 10 feet 6 inches from the center of gravity, an upward or downward thrust of about 80 pounds will be exerted upon it solely as the result of pounds will be exerted upon it solely as the result of sizes found on most machines, it may be stated as a. sizes found on most machines, it may be stated as $a$.
rough approximation that the loading represented by rough approximation that the loading represented by
such a force is approximately of the same order as that normally carried by the main planes themselves. This gyrostatic reaction on the part of a rotating mass can be very easily demonstrated by anyone who cares to spend a few cents and a couple of hours in cares to bend a few cents and a couple of hours in and model such as is illustrated in Fig. 1. The model represents a rough approximation to a monoplane, cut out of a piece of
stiff cardboard. It need not be accurate in any way so stiff cardboard. It need not be accurate in any way so
long as it has a couple of wings and some sort of a tail. long as it has a couple of wings and some sort of a tail.
Its head is stiffened by a piece of board, $A$, in which Its head is stiffened by a piece of board, $A$, in which
is cut a square opening to receive a gyroscope to such as may be bought for 25 cents at any toy shop. The frame of this top must be clamped down to the board so that the axis of the spinning-shaft lies fore and aft and the top is quite free to rotate. The two wings of the model are stiffened by attaching a strip wings of the model are stiffened by attaching a strip
of thin wood underneath them in the form of a main transverse spar, and the extremities are whittled don $n$ into a round section so that they can be mounted freely in the upright supports of the frame, which is made. as shown in the sketch. To the head and the tail of the model a piece of bent cardboard must be attached, as shown at $C$, in order to bring the center of gravity somewhat under the axis of suspension. The whole must then be balanced by adding a small piece of wood to one end or the other.

As long as the top is at rest, the board on which the model is mounted can be moved across the face of a table in any direction without affecting the balance of the model, but when the top is set spinning an entirely different state of affairs exists, for directly

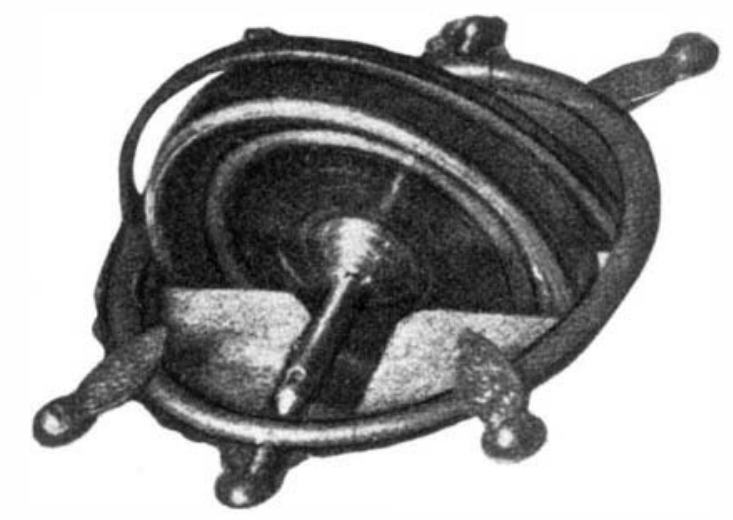

Fig. 3.-Photograph Illustrating How an Ordinary Toy Gyroscopic Top Can be Mounted in the Model Shown in the Sketch.

the board is turned so as to make the model reproduce the action of steering to the right or left, the gyroscopic reaction will immediately cause the model as a whole to dive or tilt.

Possibly some may think that the quantitative analysis made above represents an exaggerated case, but lately some flying machines have been fitted with 100-horse-power motors weighing 100 hilograms. Furthermore, a turn at right angles in three seconds is

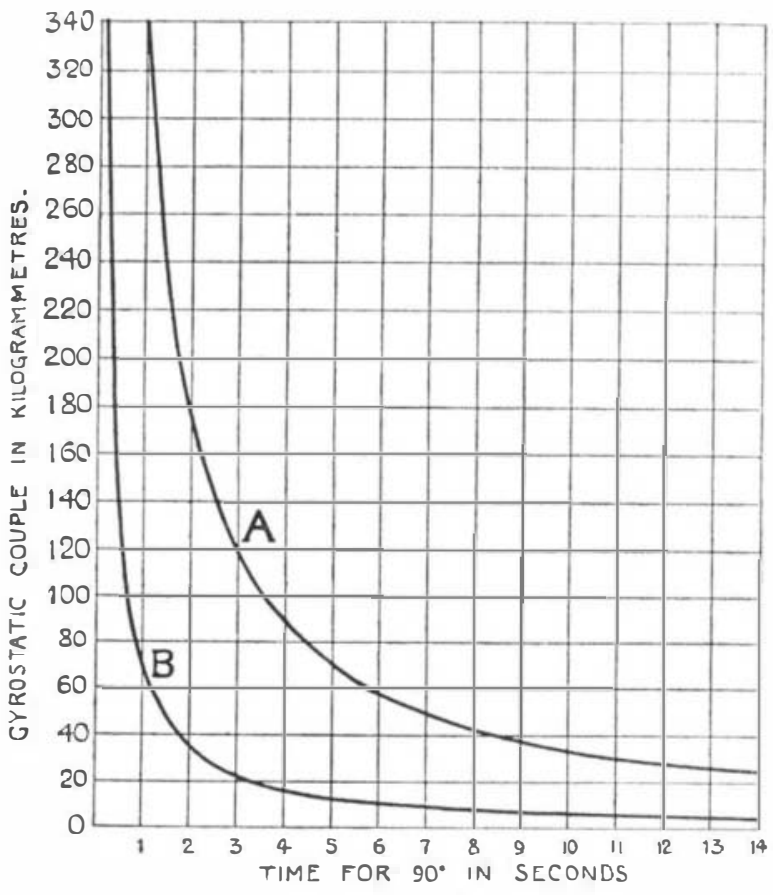

Fig. 4-Chart Showing the Magnitude of the Gyrostatic Couple Caused by Changing the Direction of Flight to a Line at Right Angles to the Origina Path in the Time Stated. Graph $A$ Shows Vaiue for a Rotary Engine Weighing 100 Kilogram Operating a Propeller Weighing 10 Kilograms While Graph $B$ shows Values for a Stationary Engine Driving a Propeller Weighing 5 Kilo grammes (11.03 Pounds) Also at 1200 Revolutions per Minute.

nothing exceptional, for, as stated, Wilbur Wright often made such a turn in one second, and if we calculate the gyrostatic couple, for such a quick movement, on similar lines as above, we find that this cou ple amounts to 340 kilogram meters (2,459 foot pounds).

On the other hand there is one aspect of the problem which makes it necessary to consider the worst case 
Even though a pilot may take precautions to the extent of not voluntarily turning too quickly, nevertheless in windy weather the action of the wind itsel may force the machine suddenly to change its position and thus bring these very gyrostatic forces into exaggerated effect.

Having explained the danger, it would be incomplete not to discuss its remedy, which is that of duplicating the rotating members and making them rotate in opposite directions. It does not at present seem feasible to do this with rotary engines, but with stationary engines, where only the propellers create a gyrostatic effect of any appreciable magnitude, it 1 not difficult to employ two propellers rotating in opposite directions. This has been done by the Brother Wright, and there is very little doubt in my mind that they have actually accomplished maneuvers in the air with their machine of such intricacy as could not with their machine of such of neutralizing* gyroscopic action has not been taken No other pilot that I have ever seen ever executed turns and figures of eight with the same rapidity' a Wilbur Wright was at one time accustomed to carry out them on his own twin-screw biplane, and feel convinced that it is not only in the relative skil of the pilots that we must seek for the reason.

Those who may be inspired to make the little model that 1 discribed above, may conceivably be interested in having a mental conception of the mechanics of gyrostatic action, and although the subject is one of great intricacy in its complete elucidation there is no reason whatever why anyone should not be able to carry away a very clear idea of the actions and reactions that bring about the particular phenomena under consideration. For this purpose I have pre pared a little diagram (Fig. 2) showing a spinning disk and have supposed that the spindle $a, b$, of the disk, and have supposed that the spindle, $a, b$, of the disk has been forcibly rocked over in the direction. $x$ so that it is caused to assume the inclined position i lustrated. It is our purpose to investigate the forces called into being at the moment that this displacement tends to commence. Across the face of the disk I hav drawn a diameter, $o, q$, representing the axis abou which the spindle is turning in the direction, $x$, an from the point 0 are drawn two tangential lines, $0, r$ and $0, s$, the first named representing the direction in which a that ficle of the disk at 0 , was traveling befor the spindle moved, while $o, s$ represents the direction of this same particle after the spindle has been displaced. Now, it is very obvious that this change in directior of the movement of the particle can only have been brought about by the application of an upward force, and by Newton's laws it is equally obvious that the action has been resisted by a downward action, which I have represented by the line, $o$, $t$, on the diagram. At the other extremity of the diameter, $q$, the reaction will, for the same reason, be upward. These reactions constitute a couple, called the preressional couple, that tends to turn the disk about a line, $n, p$, at right angles to the line, $a, g$ and to the line, $a, b$ or axis of rotation of the disk. The partiine, $a, b$, axis of rotation of the disk. The partidiameter at right angles to $o, q$, do not give rise to gyrostatic reaction, because the new direction of their movement has remained parallel to their original path. From this diagram it should be quite evident that two similar disks rotating in opposite directions, but upon the same axis, would completely neutralize each other's gyrostatic reaction.

In order further to assist any readers who may be interested in this important subject, I have prepared a chart (Fig. 4) showing by means of a graph, $A$, the gyrostatic couple produced by the masses taken in the special case already discussed. The base line of the diagram represents the time taken to turn through 90 degrees while the vertical scale represents the gyrostatic couple in kilogram-meters. The lower curve, $B$, shows values for an ordinary stationary motor fitted with a single light propeller, weighing 5 kilograms, and having a diameter of 2.40 meters ( 7.87 feet), making 1,200 revolutions per minute.

\section{Simple Household Inventions ${ }^{\dagger}$}

NEW SWEEPING MACHINES.

THF last Lépine exhibition contained several interesting new devices for sweeping carpets and scrubbing floors. The simplest is the Abor broom with detachable handle (Fig. 1). This broom is designed to replace the cheap round broom of rice straw which is com monly used in France and which after a few day use becomes one-sided and almost worthless. The Abor broom is also made of rice straw, but it is fla and the straw is securely fastened in a metal head,

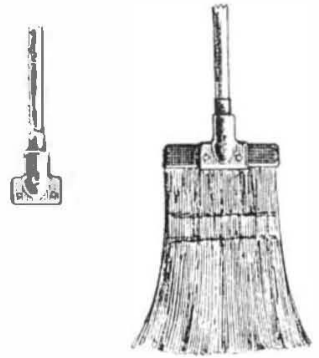

Fig. 1.-Abor Detachable Broom.

which is attached to the handle by a screw, so that when the brush is worn out, it can be replaced with out renewing the handle.

The Astra brush (Fig. 2) is a carpet sweeper re duced to its simplest form. Two cylindrical brushe duced to its simplest form. Two cylindrical brushes
$B$, geared to two heavy fly-wheels $V$, at the lower end $B$, geared to two heavy fly-wheels $V$, at the lower end
of a long metal tube, are turned by a crank and whee at the upper end, by means of an endless cord inside

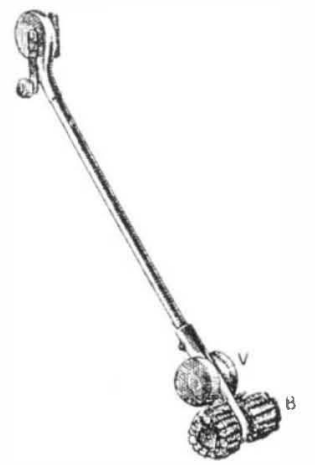

Fig. 2.-Astra Brush.

the tube. The brushes are easily detached and re placed by softer or harder brushes, as the work may require.

The Baranger mechanical brush (Fig. 3) is mor complicated and produces an effect identical with that of a hand scrubbing brush. Two flat brushes, $A$ and $B$, are mounted in a frame, to which a reciprocating

* We would point out that when the rotating members ar oot located on the same axis, their individual gyrostatic forces, heing of opposite signs, create a couple that stresses the frame work of the machine between the axes in question. Thus, for
esample, the gyrostatic forces of the two propellers on Wrigle, biplane must tend to twist the structure, which is course, sufficiently strong to prevent damage, although algebraic sum of the two forces, as effecting the control of the machine, is zero. - The Editor of Flight.

$\dagger$ Compiled from La Nature.

\section{Clever Novelties from Abroad}

movement is given by a rod connected with an eccen tric. The front brush, $B$, is much wider than the following brush, $A$, the function of which is to clean depressions that the larger brush has passed over. The shaft of the eccentric, which also carries two heavy fly-wheels and a toothed pinion, is turned by means of a bicycle chain which passes over a sprocket

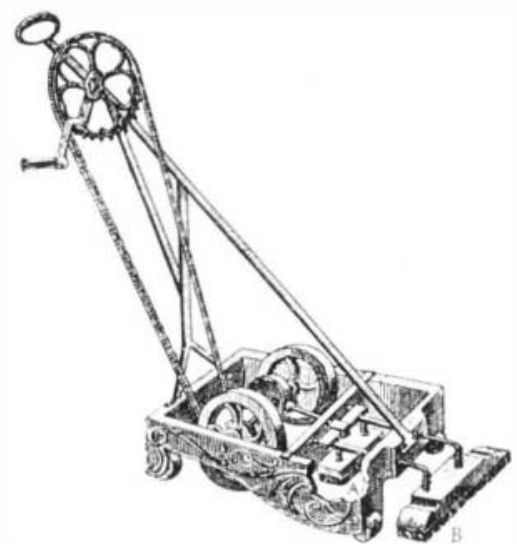

Fig. 3.-Baranger Mechanical Brush.

wheel, provided with a crank, at the end of the handle The brushes may also be operated by a small electric motor, mounted on the wheeled frame.

Electric power is employed exclusively in the Bruy'ère electric sweeper (Fig. 4), which is designed primarily for cleaning large floors in public buildings An electric motor, $E$, connected with the mains by a

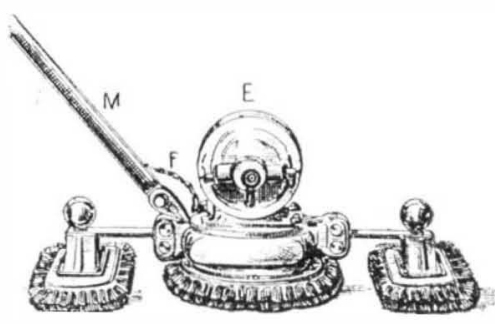

Fig. 4.-Bruyère Electric Sweeper

flexible cable, $F$, drives a central rotary brush, the shaft of which carries an eccentric that communicates a reciprocating movement to a flat brush on each side. The machine can be moved with very little effort by means of the handle $M$, and 4,000 square feet of floor means of the handle $M$, and 4,000 square feet of floor of current.

\section{NEW CHIMNEY DRAFT GAGE.}

For the purpose of measuring the slight barometric depressions which occur in factory chimneys, it is customary to employ manometers of speccial construction and great sensitiveness. One of the most frequently used devices consists of a bent tube with one branch vertical and the other nearly horizontal (Fig. 5). A small quantity of colored liquid is placed in the bend of the tube, of which the vertical branch communicates with the chimney and the inclined branch with the external atmosphere. The variations of level in either branch rarely exceed 2 inches, but the movement of the liquid surface along the inclined branch is very much greater, so that the variations of pressure can be easily and accurately read from a scale attached to the tube.

This and the other devices in use are bulky and easily broken. An American inventor has devised the expedient of coiling the inclined branch of the tube round the vertical branch in the form of a helix, thus constructing the strong and compact apparatus shown in Fig. 6. The scale follows the helix and, in order to read it throughout, the apparatus must be visible

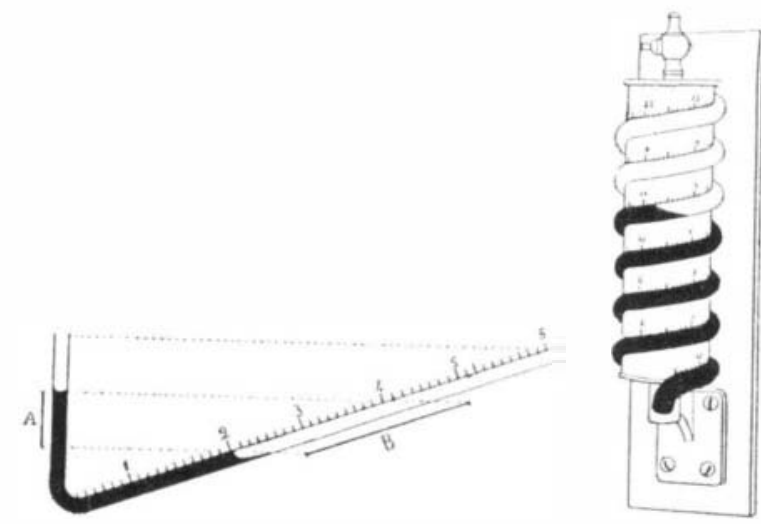

Figs. is and 6.

New Chimney uraft Gage.

from all sides. Even this slight inconvenience could be eliminated by making the rear portions of the outer tube horizontal, but this would be useless. In practice, it is sufficient for the regulation of the draft to note whether the end of the column of liquid is at the left or right side of a visible segment of the tube or in the invisible segment between two visible segments, the scales of which can be read.

IDEAL PENCIL SHARPENER.

The new Ideal pencil sharpener differs from other small devices of its class in the excellent quality of its two steel blades, which produce a very fine point, and in the fact that it is inclosed in a little box of sheet copper into which the pencil point is inserted through a lateral opening. The box collects the chips
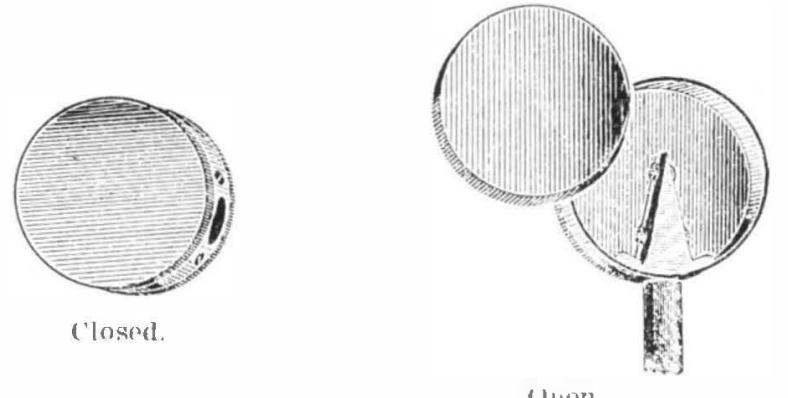

(1) (1)

Fig. 7-Ideal Pencil Sharpener.

and dust, thus protecting the carpet and the operator's fingers from soiling. The box has a removable cover and can be emptied and cleaned whenever it is convenient to do so. 